\title{
Front Matter: Volume 10291
}

, "Front Matter: Volume 10291," Proc. SPIE 10291, Materials Characterization and Optical Probe Techniques: A Critical Review, 1029101 (30 July 1997); doi: 10.1117/12.2284219

SPIE Event: Optical Science, Engineering and Instrumentation '97, 1997, San Diego, CA, United States 


\section{Contents}

\section{SESSION 1}

Mid- and near-infrared spectroscopy of polymers: time-resolved studies and remote sensing applications [CR69-01]

S. Okretic, N. Völkl, H. W. Siesler, Univ. of Essen (FRG)

Four-wave mixing: applications to fundamental research and photonics technology [CR69-02]

P. N. Prasad, SUNY/Buffalo

Characterization of polymers in solution with light scattering [CR69-03]

E. H. Nordmeier, Univ. Osnabrück (FRG)

Mössbauer spectroscopy of iron containing polymers [CR69-05]

H. Franke, Univ. Duisburg (FRG)

\section{SESSION 2}

UV-vis spectroscopy as an analytical tool for the characterization of polymers [CR69-06]

G. Manivannan, Surfacine Consumer Products LIC

Laser atomic fluorescence characterization of materials [CR69-07]

S. B. Mirov, A. Yu. Dergachev, Univ. of Alabama/Birmingham

146 Dielectric spectroscopy as an analytical technique for the characterization of polymeric solids [CR69-09]

G. J. Pratt, Univ. of Melbourne (Australia); M. J. A. Smith, Univ. of Warwick (UK)

\section{SESSION 3}

175 SEM techniques for materials characterization [CR69-10]

J. Beauvais, D. Drouin, R. Gauvin, Univ. de Sherbrooke (Canada)

200 Review of transmission electron microscopy for the characterization of materials [CR69-11]

R. Gauvin, Univ. de Sherbrooke (Canada)

Materials analysis using high-energy ion scattering [CR69-12]

E. J. Knystautas, Univ. Laval (Canada)

Characterization of optical materials using Auger electron spectroscopy [CR69-13]

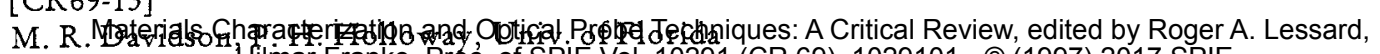
Hilmar Franke, Próc. of SPIE Vol. 10291 (CR 69), 1029101 - (c) (1997) 2017 SPIE CCC code: $0277-786 \mathrm{X} / 17 / \$ 18 \cdot$ doi: $10.1117 / 12.2284219$

$\checkmark$

Proc. of SPIE Vol. 10291 1029101-1 
280 Surface analysis with scanning probe microscopy [CR69-14]

T. G. Ruskell, National Institute of Standards and Technology

\section{SESSION 4}

307 Holographic characterization of optical materials [CR69-15]

R. A. Lessard, Univ. Laval (Canada)

326 Photothermal sensing techniques for thin film characterization [CR69-16]

Z. L. Wu, Eastern Michigan Univ.

357 Core level spectroscopy for surface analysis [CR69-18]

F. J. Himpsel, R. Treusch, Univ. of Wisconsin/Madison; I. Jimenez,

A. F. Jankowski, D. G. J. Sutherland, L. J. Terminello, Lawrence Livermore

National Lab.; C. Heske, R. C. C. Perera, D. K. Shuh, W. M. Tong,

J. H. Underwood, Lawrence Berkeley National Lab.; J. A. Carlisle, Virginia

Commonwealth Univ.; T. A. Callcott, J. J. Jia, Univ. of Tennessee/Knoxville;

D. L. Ederer, Tulane Univ.; D. M. Gruen, A. R. Krauss, D. C. Zuiker, Argonne

National Lab.; G. L. Doll, Timkin Research

\section{SESSION 5}

377 EPR and ENDOR characterization of nonlinear optical materials [CR69-19]

L. E. Halliburton, West Virginia Univ.

398 Ellipsometry as a characterization technique [CR69-20]

V.-V. Truong, Univ. de Moncton (Canada); N. Le-quang, Nanoptix Inc. (Canada)

423 SIMS characterization of optical materials [CR69-22]

F. Caccavale, Univ. di Padova (Italy)

\section{SESSION 6}

463 Characterization of polymeric films by integrated optics [CR69-23]

J. D. Swalen, Univ. of California/Santa Cruz

Electric-field-induced second harmonic generation [CR69-24]

F. Kajzar, CEA-LETI (France)

501 Z-scan technique for nonlinear materials characterization [CR69-25]

E. W. Van Stryland, CREOL/Univ. of Central Florida; M. Sheik-Bahae, Univ. of New Mexico

Characterization of polymers and particles with the analytical ultracentrifuge [CR69-26]

H. H. Cölfen, Max Planck Institute of Colloids and Interfaces (FRG) 


\title{
Conference Committee
}

\author{
Conference Chairs
}

Roger A. Lessard, Université Laval (Canada)

Hilmar Franke, Universität Duisburg (FRG)

Session Chairs

1 Roger A. Lessard, Université Laval (Canada)

2 Hilmar Franke, Universität Duisburg (FRG)

3 Vo-Van Truong, Université de Moncton (Canada)

4 François Kajzar, CEA-Technologies Avancées (France)

5 Emile J. Knystautas, Université Laval (Canada)

6 Robert A. Fisher, RA Fisher Associates

vii

Proc. of SPIE Vol. 10291 1029101-3

Downloaded From: https://www.spiedigitallibrary.org/conference-proceedings-of-spie on 26 Apr 2023 Terms of Use: https://www.spiedigitallibrary.org/terms-of-use 